\title{
Impact of node inter-domain movement on MANETs performance
}

\begin{abstract}
Packet delivery ratio (PDR) percentage is one of the important network performance indicators in MANETs. In general, the PDR value degrades as speed of the node increases and coupled with high mobility or constant movement. As more nodes move at high speed, more broken path or link breakage occur and thus, more packets will be dropped. Interestingly, PDR rate has also been used to detect packet drop or black hole attack in the network. Thus, the packet drop activity may due to either the broken path process itself or deliberate drop by malicious nodes. Validating the packet drop action itself is imperative in reducing the false positive rate during the attack detection. This paper studies the movements of nodes in the networks that have caused high packet drop percentage. In particular, we investigate the inter-domain movement since it has substantial effect on the packet drop percentage. To the best of our knowledge, this is the first work that studies such relationship. The results on the overall network show that the high number of inter-domain movement may not necessarily contribute significantly to the packet drop percentage. However, when focus is on the inter-domain movement of the critical nodes, we yield consistent results. The proposed monitoring approach is also energy efficient as it reduces the need to monitor other large number of nodes insignificant movements.
\end{abstract}

Keyword: Inter-domain movement; Mobile ad hoc networks; Packet drop validation 